\title{
THE LEAST-LUMINOUS GALAXY: SPECTROSCOPY OF THE MILKY WAY SATELLITE SEGUE 1
}

\author{
Marla Geha $^{1}$, Beth Willman ${ }^{2}$, Joshua D. Simon ${ }^{3}$, Louis E. Strigari ${ }^{4,7}$, Evan N. Kirby ${ }^{5}$, David R. Law ${ }^{3}$, and Jay Strader 6 \\ ${ }^{1}$ Astronomy Department, Yale University, New Haven, CT 06520, USA; marla.geha@yale.edu \\ ${ }^{2}$ Haverford College, Department of Physics, 370 Lancaster Avenue, Haverford, PA 19041, USA \\ ${ }^{3}$ Department of Astronomy, California Institute of Technology, 1200 E. California Blvd., MS 105-24, Pasadena, CA 91125, USA \\ ${ }^{4}$ Kavli Institute for Particle Astrophysics \& Cosmology, Physics Department, Stanford University, Stanford, CA 94305, USA \\ ${ }^{5}$ UCO/Lick Observatory, University of California, Santa Cruz, 1156 High Street, Santa Cruz, CA 95064, USA \\ ${ }^{6}$ Harvard-Smithsonian CfA, 60 Garden St., Cambridge, MA 02144, USA \\ Received 2008 August 3; accepted 2008 October 10; published 2009 February 24
}

\begin{abstract}
We present Keck/DEIMOS spectroscopy of Segue 1, an ultra-low-luminosity $\left(M_{V}=-1.5_{-0.8}^{+0.6}\right)$ Milky Way satellite companion. While the combined size and luminosity of Segue 1 are consistent with either a globular cluster or a dwarf galaxy, we present spectroscopic evidence that this object is a dark matter-dominated dwarf galaxy. We identify 24 stars as members of Segue 1 with a mean heliocentric recession velocity of $206 \pm 1.3 \mathrm{~km} \mathrm{~s}^{-1}$. Although Segue 1 spatially overlaps the leading arm of the Sagittarius stream, its velocity is $100 \mathrm{~km} \mathrm{~s}^{-1}$ different from that predicted for recent Sagittarius tidal debris at this position. We measure an internal velocity dispersion of $4.3 \pm 1.2 \mathrm{~km} \mathrm{~s}^{-1}$. Under the assumption that these stars are gravitationally bound and in dynamical equilibrium, we infer a total mass of $4.5_{-2.5}^{+4.7} \times 10^{5} M_{\odot}$ in the mass-follow-light case; using a two-component maximum-likelihood model, we determine a mass within $50 \mathrm{pc}$ of $8.7_{-5.2}^{+13} \times 10^{5} M_{\odot}$. These imply mass-to-light $(\mathrm{M} / \mathrm{L})$ ratios of $\ln \left(\mathrm{M} / \mathrm{L}_{V}\right)=7.2_{-1.2}^{+1.1}\left(\mathrm{M} / \mathrm{L}_{V}=1320_{-940}^{+2680}\right)$ and $\mathrm{M} / \mathrm{L}_{V}=2440_{-1775}^{+1580}$, respectively. The error distribution of the $\mathrm{M} / \mathrm{L}$ is nearly lognormal, thus Segue 1 is dark matter-dominated at a high significance. Although we cannot rule out the possibility that Segue 1 has been tidally disrupted, we do not find kinematic evidence supporting tidal effects. Using spectral synthesis modeling, we derive a metallicity for the single red giant branch star in our sample of $[\mathrm{Fe} / \mathrm{H}]=-3.3 \pm 0.2$ dex. Finally, we discuss the prospects for detecting gamma rays from annihilation of dark matter particles and show that Segue 1 is the most promising satellite for indirect dark matter detection. We conclude that Segue 1 is the least luminous of the ultra-faint galaxies recently discovered around the Milky Way, and is thus the least-luminous known galaxy.
\end{abstract}

Key words: galaxies: dwarf - galaxies: individual (Segue 1) - galaxies: kinematics and dynamics - Local Group

Online-only material: color figures, machine-readable table

\section{INTRODUCTION}

The discovery of "ultra-faint" dwarf spheroidal (dSph) galaxies around the Milky Way has revolutionized our understanding of dwarf galaxies and their prevalence in the universe. These newly discovered satellites, with total absolute magnitudes fainter than $M_{V}=-8$, have all been found in the Sloan Digital Sky Survey (SDSS) via slight statistical overdensities of individual stars (Willman et al. 2005a, 2005b; Zucker et al. 2006a, 2006b; Belokurov et al. 2006a, 2007; Sakamoto \& Hasegawa 2006; Irwin et al. 2007; Walsh et al. 2007). These objects provide important clues to galaxy formation on the smallest scales (Madau et al. 2008; Ricotti et al. 2008) and substantially alleviate the discrepancy between the observed mass function of Milky Way satellites and that predicted by standard Lambda Cold Dark Matter models (Tollerud et al. 2008; Simon \& Geha 2007, hereafter SG07). Strigari et al. (2008b) note that the ultrafaint dSphs have high central dark matter densities and are good candidates for indirect dark matter detection via gamma-ray emission by particle annihilation. Future wide-field surveys that improve on the sky coverage and photometric depth of the SDSS are likely to discover many additional ultra-faint Milky Way satellites in the coming years (Koposov et al. 2008; Walsh et al. 2009).

While the total luminosities of the ultra-faint satellites are comparable to globular clusters, spectroscopic studies for the

\footnotetext{
7 Hubble Fellow.
}

majority of the newly discovered objects firmly suggest that these objects are dark matter-dominated dwarf galaxies (Kleyna et al. 2005; Muñoz et al. 2006; Martin et al. 2007, SG07). The mass-to-light $(\mathrm{M} / \mathrm{L})$ ratios for all the ultra-faint dSphs are $\mathrm{M} / \mathrm{L}_{V}>100 M_{\odot} / L_{\odot}$, with several systems approaching $1000 M_{\odot} / L_{\odot}$, assuming mass follows light. Strigari et al. (2008a) loosened this constraint, confirming the high M/Ls and finding a tight anticorrelation between $\mathrm{M} / \mathrm{L}$ and luminosity such that all the Milky Way dwarfs are consistent with having a common dark matter mass of $\sim 10^{7} M_{\odot}$ within their central $300 \mathrm{pc}$. A theoretical understanding of the physics that sets the mass-luminosity relation will provide insight into the formation of galaxies at the smallest scales.

Further evidence that the ultra-faint satellites are indeed galaxies comes from metallicity measurements. The ultra-faint satellites are the most metal-poor known stellar systems $([\mathrm{Fe} / \mathrm{H}]$ $<-2$ ) and show internal metallicity spreads up to $0.5 \mathrm{dex}$ in several objects (SG07). This is in contrast to Milky Way globular clusters which are, on average, more metal rich and show little to no internal metallicity spread (e.g., Pritzl et al. 2005). In further contrast to globular clusters, the ultra-faint dwarfs also follow the luminosity-metallicity relationship established by brighter Milky Way dwarf galaxies (Kirby et al. 2008b). Thus, both the kinematics and composition of the ultra-faint satellites strongly argue that these objects are dark matter-dominated galaxies.

The combined size and luminosity of the spectroscopically confirmed dSphs in the Milky Way are well separated from 

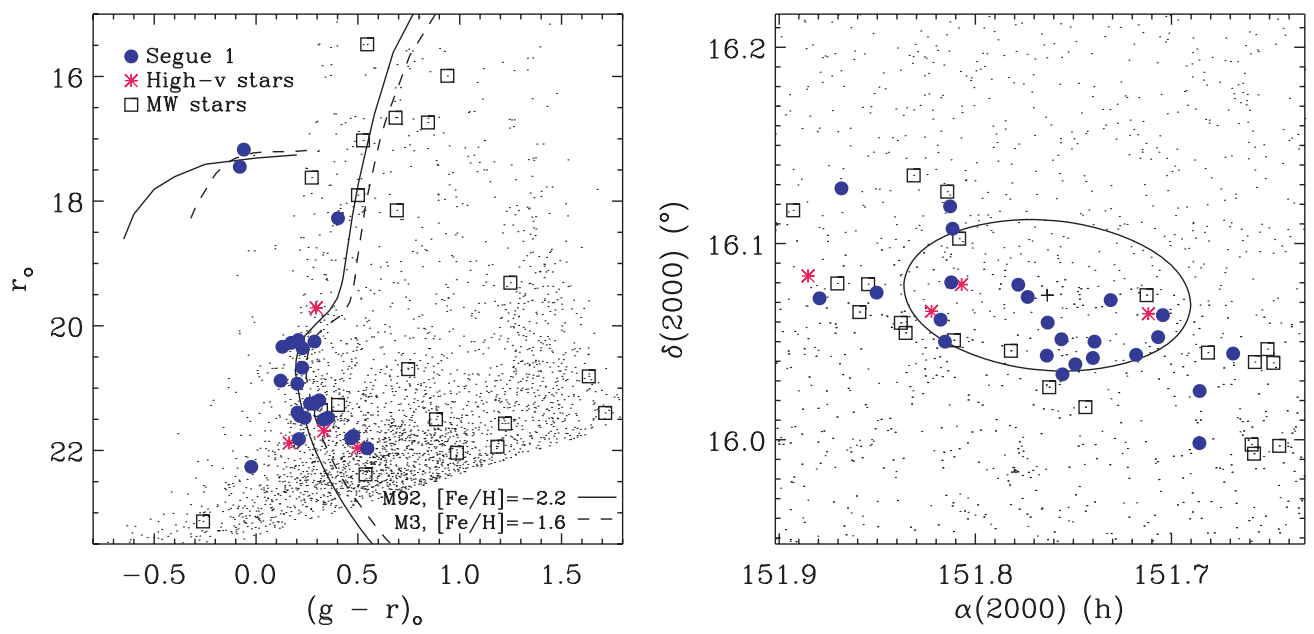

Figure 1. Left: Color-magnitude diagram of all stars (small black points) within $30^{\prime}$ of the center of Segue 1 from SDSS DR $6 g$-and $r$-band photometry. The larger symbols indicate stars with measured Keck/DEIMOS velocities: the solid blue circles fulfill our requirements for membership in Segue 1, the red asterisks are higher velocity stars and the open squares are foreground Milky Way stars. Two fiducial isochrone are shown shifted to the distance of Segue 1: M92 ([Fe/H] $=-2.3$, solid line) and M3 ([Fe/H] $=-1.6$, dashed line). Right: Spatial distribution of stars near Segue 1. The solid ellipse is the half-light radius of Segue 1 as measured by Martin et al. (2008).

(A color version of this figure is available in the online journal.)

globular clusters: at a given luminosity dwarf galaxies have larger sizes and are thus less compact (Belokurov et al. 2007; Martin et al. 2008). However, the three faintest SDSS discoveries, Segue 1, Willman 1 and Bootes II, are all in a region that overlaps with globular clusters. Studying these extreme systems should provide important insight to dSphs, and the difference between dwarfs and star clusters, at all luminosities. Of these three objects, only Willman 1 has published kinematics (Martin et al. 2007). Because the systemic velocity of Willman 1 is similar to that of the foreground Milky Way stars, possible contamination in the kinematic sample make it difficult to assess whether this object is a dwarf or globular cluster (Siegel et al. 2008, B. Willman et al. 2009, in preparation). Here, we present the first spectroscopic study of an even lower luminosity system, Segue 1 . The systemic velocity of Segue 1 is far removed from the Milky Way foreground and thus should be a cleaner object to study the properties of the least-luminous ultra-faint systems.

Segue 1 was discovered by Belokurov et al. (2007) as an overdensity of resolved stars in the SDSS located at $\left(\alpha_{2000}, \delta_{2000}\right)$ $=(10: 07: 03,+16: 04: 25)=(151.763,16: 074)$. Via isochrone fitting, these authors estimate a distance of $23 \pm 2 \mathrm{kpc}$ and an absolute luminosity of $M_{V} \sim-3 \pm 0.6$. Martin et al. (2008) recently revised the luminosity of Segue 1 to $M_{V}=-1.5_{-0.8}^{+0.6}$ using a more robust method to estimate flux in systems with small numbers of observable stars. While the possibility of tidal tails and/or tidal distortion of Segue 1 was found in the initial SDSS analysis, deeper imaging and more thorough simulations suggest that these features can be explained via Poisson scatter of the few bright stars in this system (Martin et al. 2008). Segue 1 has no detected gas content, with an observed HI gas mass limit of less than $13 M_{\odot}$ (Putman et al. 2008). This limit is consistent with other dSphs around the Milky Way in which any gas has been presumably removed via ram pressure stripping or used up via tidally induced star formation Mayer et al. (2006).

Belokurov et al. (2007) note that Segue 1 is spatially superimposed on the leading arm of the Sagittarius stream. Because it has a similar luminosity and size as the most diffuse globular cluster, they proposed that Segue 1 is a globular cluster formerly associated with the Sagittarius dSph. Spectroscopy of member stars in Segue 1 is required to test this hypothesis and answer the crucial question of whether or not this intrinsically faint stellar system is truly a globular cluster (i.e., a stellar system with a single stellar population with no dark matter). Here, we present Keck/DEIMOS multiobject spectroscopy for individual stars in the vicinity of Segue 1, identifying 24 stars as members of Segue 1.

This paper is organized as follows: in Section 2, we discuss target selection and data reduction for our Keck/DEIMOS spectroscopy. In Section 3, we discuss the spectroscopic results including estimates of the velocity dispersion, mass, $M / L$, and metallicity. In Section 4, we examine the spatial and kinematic position of Segue 1 relative to the Sagittarius stream. In Section 5, we note that Segue 1 may be a good target for indirect detection of dark matter. Finally, in Section 6, we discuss Segue 1 in context of the Milky Way dSph population.

Throughout the analysis, we use the photometric properties of Segue 1 as derived by Martin et al. (2008) of $M_{V}=-1.5_{-0.8}^{+0.6}$ (i.e., the $1 \sigma$ magnitude limits are $M_{V}=-0.9$ and -2.3 ) and $r_{\text {eff }}=4.4_{-0.6}^{\prime+1.2}=29_{-5}^{+8}$ pc. We also assume a fixed reddening to Segue 1 based on the Schlegel et al. (1998) value of $E(B-V)$ $=0.032 \mathrm{mag}$. We list these and other key parameters in Table 1 .

\section{DATA}

\subsection{Target Selection}

Targets were selected for spectroscopy based on gri photometry of Segue 1 from the SDSS DR6 public database (Adelman-McCarthy et al. 2008). As discussed in SG07, we set the target priorities to preferentially observe stars with a high likelihood of being Segue 1 members. Using the theoretical isochrones of Clem et al. (2008) and Girardi et al. (2004), we chose targets whose color and apparent magnitudes minimize the distance from the best-fitting Segue 1 isochrone. The highest priority targets were those located within $0.1 \mathrm{mag}$ of the red giant branch (RGB) tracks, or within 0.2 mag of the horizontal branch, with additional preference being given to brighter stars (Figure 1). Stars farther from any of the fiducial sequences were classified as lower-priority targets. We designed the slitmask so as to maximize the number of high-priority targets: a total of 59 targets were placed on the Segue 1 mask, 26 of which were in 
Table 1

Summary of Observed and Derived Quantities for Segue 1

\begin{tabular}{cccc}
\hline \hline Row & Quantity & Units & Segue 1 \\
\hline$(1)$ & R.A. & $\mathrm{h}: \mathrm{m}: \mathrm{s}$ & $10: 07: 03.2 \pm 1.7^{\mathrm{s}}$ \\
$(2)$ & Decl. & $:^{\prime}:^{\prime \prime}$ & $+16: 04: 25 \pm 15^{\prime \prime}$ \\
$(3)$ & $E(B-V)$ & $\mathrm{mag}$ & 0.032 \\
$(4)$ & Dist & $\mathrm{kpc}$ & $23 \pm 2$ \\
$(5)$ & $M_{V, 0}$ & $\mathrm{mag}$ & $-1.5_{-0.8}^{+0.6}$ \\
$(6)$ & $L_{V, 0}$ & $L_{\odot}$ & 340 \\
$(7)$ & $\epsilon$ & & $0.48_{-0.13}^{+0.10}$ \\
$(8)$ & $\mu_{V, 0}$ & $\mathrm{mag} \mathrm{arcs}^{-2}$ & $27.6_{-0.7}^{+1.0}$ \\
$(9)$ & $r_{\mathrm{eff}}$ & $\mathrm{pc}_{-0.6}$ & $29_{-5}^{+8}$ \\
$(10)$ & $r_{\mathrm{eff}}$ & $\mathrm{km} \mathrm{s}^{-1}$ & $206.4 \pm 1.3$ \\
$(11)$ & $v$ & $\mathrm{~km} \mathrm{~s}^{-1}$ & $114 \pm 2$ \\
$(12)$ & $v_{\mathrm{GSR}}$ & $\mathrm{km} \mathrm{s}^{-1}$ & $4.3 \pm 1.2$ \\
$(13)$ & $\sigma$ & $M_{\odot}$ & $4.3_{-2.5}^{+4.7} \times 10^{5}$ \\
$(14)$ & $\mathrm{Mass}$ & $M_{\odot} / L_{\odot}$ & $1340_{-990}^{+4340}$ \\
$(15)$ & $\mathrm{M} / \mathrm{L}$ & $\mathrm{dex}$ & $-3.3 \pm 0.2$ \\
$(16)$ & {$[\mathrm{Fe} / \mathrm{H}]$} & &
\end{tabular}

Notes. Columns (1)-(2) and (5)-(10) taken from the SDSS photometric analysis of Martin et al. (2008). Column (3) from Schlegel et al. (1998) and (4) from Belokurov et al. (2007). Columns (11)-(16) are derived in Section 3.

our highest priority category. Slitmasks were created using the DEIMOS dsimulator package in IRAF.

\subsection{Spectroscopy and Data Reduction}

One multislit mask was observed for Segue 1 using the Keck II 10 m telescope and the DEIMOS spectrograph (Faber et al. 2003) on the night of 2007 November 12. The mask was observed for a total of $5400 \mathrm{~s}$ through the 1200 line $\mathrm{mm}^{-1}$ grating covering a wavelength region $6400-9100 \AA$. The spatial scale is 0.12 per pixel, the spectral dispersion of this setup is $0.33 \AA$, and the resulting spectral resolution is $1.37 \AA$ (FWHM). Slitlets were 0.7 wide. The minimum slit length was $5^{\prime \prime}$ to allow adequate sky subtraction; the minimum spatial separation between slit ends was 0.'4 (3 pixels).

Spectra were reduced using a modified version of the spec $2 d$ software pipeline (version 1.1.4) developed by the DEEP2 team at the University of California-Berkeley for that survey. A detailed description of the two-dimensional reductions can be found in SG07. The final one-dimensional spectra are rebinned into logarithmic wavelength bins with $15 \mathrm{~km} \mathrm{~s}^{-1}$ per pixel.

\subsection{Radial Velocities and Error Estimates}

Radial velocities were measured by cross-correlating the observed science spectra with a set of high signal-to-noise stellar templates. The method is the same as that described in SG07 and briefly repeated here. Stellar templates were observed with Keck/DEIMOS using the same setup as described in Section 2.2 and covering a wide range of stellar types (F8 to M8 giants, subgiants, and dwarf stars) and metallicities $([\mathrm{Fe} / \mathrm{H}]=-2.12$ to +0.11 dex). We calculate and apply a telluric correction to each science spectrum by cross correlating a hot stellar template with the night sky absorption lines following the method in Sohn et al. (2007). The telluric correction accounts for the velocity error due to mis-centering the star within the 0.7 slit caused by small mask rotations or astrometric errors. We apply both a telluric and heliocentric correction to all velocities presented in this paper.

The random component of the velocity error is calculated using a Monte Carlo bootstrap method. Noise is added to each pixel in the one-dimensional science spectrum, we then recalculate the velocity and telluric correction for 500 noise realizations. The random error is defined as the square root of the variance in the recovered mean velocity in the Monte Carlo simulations. The systematic contribution to the velocity error was determined by SG07 to be $2.2 \mathrm{~km} \mathrm{~s}^{-1}$ based on repeated independent measurements of individual stars. Since we are using the same spectrograph setup and reduction methods, we assume the systematic error contribution is constant across the two runs. We add the random and systematic errors in quadrature to arrive at the final velocity error for each science measurement. Radial velocities were successfully measured for 49 of 59 extracted spectra. The median velocity error of these 49 stars is $3.6 \mathrm{~km} \mathrm{~s}^{-1}$ similar to that of SG07. The median velocity error of the 24 Segue 1 members (see below) is $5.2 \mathrm{~km} \mathrm{~s}^{-1}$ since these stars are fainter than the sample average. The majority of spectra for which we could not measure a redshift did not have a sufficient signal-to-noise ratio. The fitted velocities were visually inspected to ensure reliability. The resulting velocities and associated errors are listed in Table 2.

\section{SPECTROSCOPIC OBSERVATIONS OF SEGUE 1}

\subsection{Foreground Contamination and Membership Criteria}

In Figure 2, we identify Segue 1 as the overdensity of stars with radial velocities near $206 \mathrm{~km} \mathrm{~s}^{-1}$. We estimate possible foreground contribution below and then discuss our criteria for Segue 1 membership, which we base only on velocity.

We expect minimal contamination from foreground Milky Way stars at the position and velocity of Segue 1 . Segue 1 lies at a Galactocentric position of $(l, b)=(220.5,50.4$. According to the Besancon star count model ${ }^{8}$ of the Milky Way (Robin et al. 2003) at this Galactic position, the velocity distribution of Milky Way foreground stars peaks at a heliocentric velocity of $20 \mathrm{~km} \mathrm{~s}^{-1}$. The Besancon models include stellar contributions from the Milky Way thin and thick disk, spheroid and stellar halo. The kinematic distribution of foreground stars is roughly approximated by a Gaussian with FHWM of $35 \mathrm{~km} \mathrm{~s}^{-1}$, however the tails of the distribution extend to significantly positive and negative velocities. The percentage of Milky Way stars expected in the presumed velocity span of Segue 1, between $190<v<220 \mathrm{~km} \mathrm{~s}^{-1}$, is $2.5 \%$ of the total distribution. Thus, if we assume that all the stars with velocities less than $100 \mathrm{~km} \mathrm{~s}^{-1}$ are Milky Way foreground stars (a total of 20 stars, see Figure 2), we predict less than one foreground star in the Segue 1 velocity range.

As noted by Belokurov et al. (2007), Segue 1 is superposed on the leading arm of the Sagittarius stream, $\sim 100^{\circ}$ away from the main body of the Sagittarius dSph. Thus, a second possible source of contamination in our Segue 1 sample is stars associated with the Sagittarius stream. As discussed in Section 4 , the predicted velocity of the leading stream at this position is $v \sim-100 \mathrm{~km} \mathrm{~s}^{-1}$, very far from the radial velocity of Segue 1. While both the trailing arm and possible older wraps of the Sagittarius stream may be present at this position, both components would have much wider velocity distributions than Segue 1. We conclude that there is no contamination from Sagittarius stream stars in the Segue 1 velocity window. Finally, there are four stars at higher velocities $\left(v \sim 300 \mathrm{~km} \mathrm{~s}^{-1}\right)$ that do not appear to be associated with Segue 1 as a result of the $100 \mathrm{~km} \mathrm{~s}^{-1}$ velocity difference which we discuss in Section 4.1.

\footnotetext{
8 http://model.obs-besancon.fr
} 

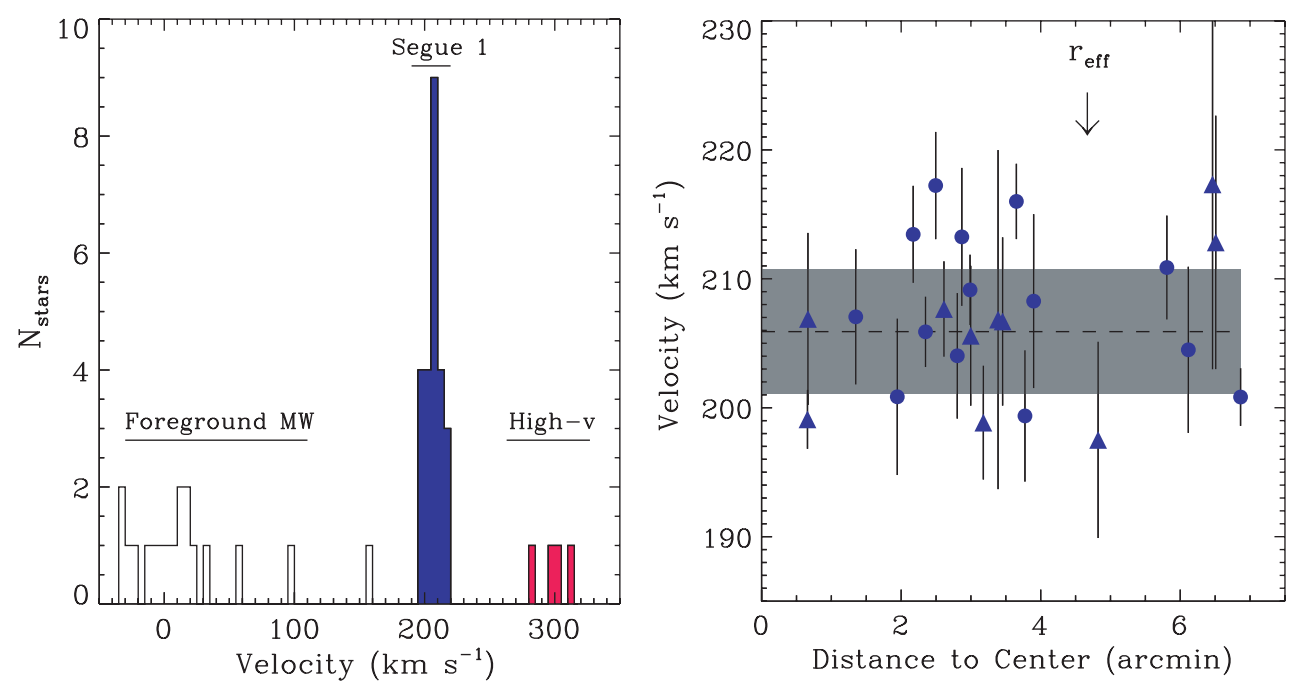

Figure 2. Left: Keck/DEIMOS velocity histogram for all stars in our sample; velocities are corrected to the heliocentric frame. We identify Segue 1 as the velocity peak near $v=206 \mathrm{~km} \mathrm{~s}^{-1}$. The stars with less positive velocities are identified as foreground Milky Way, the four stars with $v \sim 300 \mathrm{~km} \mathrm{~s}{ }^{-1}$ are tentatively associated with the Sagittarius stream as discussed in Section 4.1. Right: Radial distance from the center of Segue 1 plotted against heliocentric velocity. The stars to the east of the galaxy center are plotted as triangles, the stars to the west are plotted as squares. We indicate the effective half-light radius $\left(r_{\text {eff }}\right)$, the mean systemic velocity of the system (black dashed line), and velocity dispersion (gray-shaded region).

(A color version of this figure is available in the online journal.)

Since the expected contamination from both foreground Milky Way stars and the Sagittarius stream is low in the velocity region of Segue 1, our criteria for Segue 1 membership are simple: we assign membership based only on velocity. Stars with radial velocities between $190<v<220 \mathrm{~km} \mathrm{~s}^{-1}$ are considered members of Segue 1. This cut provides 24 member stars. The nearest nonmembers in our spectroscopic sample are at $v=155 \mathrm{~km} \mathrm{~s}^{-1}$ and $v=281 \mathrm{~km} \mathrm{~s}^{-1}$, so different velocity cuts would identify the same set of members.

The color-magnitude distribution of kinematically selected Segue 1 members is shown in Figure 1. We plot fiducial sequences for the globular clusters $\mathrm{M} 92([\mathrm{Fe} / \mathrm{H}]=-2.3)$ and M3 $([\mathrm{Fe} / \mathrm{H}]=-1.6)$. These ridge lines are based on those of Clem et al. (2008) in $g^{\prime}-r^{\prime}$, converted to $g-r$ using the transformations of Rider et al. (2004) and shifted to the distance of Segue $1(23 \mathrm{kpc})$. These fiducials are well matched to the kinematic sample. In particular, the spectra of the two bright blue stars $(r \sim 17.5, g-r \sim-0.1)$ show strong broad absorption lines of the Paschen series and narrow Ca II triplet lines consistent with being horizontal-branch stars. The position of these two stars is also well matched to the metal-poor horizontal-branch isochrones at the distance of Segue 1.

\subsection{Velocity Dispersion}

We measure the mean velocity and velocity dispersion of Segue 1 using the maximum-likelihood method described by Walker et al. (2006). This method assumes that the observed velocity dispersion is the sum of the intrinsic galaxy dispersion and the dispersion produced by measurement errors. Fitting the full Segue 1 sample based on the 24 member stars identified above, we find a mean heliocentric velocity of $206.4 \pm$ $1.3 \mathrm{~km} \mathrm{~s}^{-1}$ and a velocity dispersion of $4.3 \pm 1.2$ $\mathrm{km} \mathrm{s}^{-1}$ (Figure 2). We do not find evidence for rotation in this system, however, given the small numbers of stars we cannot rule out rotation velocities on the same order as the velocity dispersion. We test this by adding a sinusoidal term to the systemic velocity, varying the amplitude and scale radius (Strigari et al. 2008a). The most likely value for the rotation amplitude is zero, with an upper $1 \sigma$ limit of $5 \mathrm{~km} \mathrm{~s}^{-1}$. While this test justi- fies the mass modeling we use with no streaming motion in the velocities, larger kinematic data sets and smaller velocity uncertainties are necessary to test more conclusively for streaming motion Segue 1.

The gray-shaded region in the right panel of Figure 2 indicates the $1 \sigma$ width of the Segue 1 velocity distribution. We note that all the member stars lie within $2.5 \sigma$ of the systemic Segue 1 velocity. The next nearest star in velocity space is over $10 \sigma$ away. We interpret this cold distribution as evidence that there are no stars currently in the process of being tidally stripped from Segue 1 (Klimentowski et al. 2007). We note here and in Section 3.4, however, that the lack of outliers is not sufficient to prove that tidal processes are not affecting our results (e.g., Muñoz et al. 2008). This interpretation also does not mean that stars have not been previously stripped from Segue 1, and still allows for the possibility that tidal interactions are currently ongoing in the dark matter component of this object. We discuss this further in Section 3.4.

\subsection{Mass and Mass-to-Light Ratio}

We calculate the dynamical mass of Segue 1 using two different methods. In both cases, we assume that Segue 1 is a relaxed, self-gravitating, spherically symmetric system with no rotational motion. We presently ignore any effects on the mass estimates due to tidal interactions between Segue 1 and the Milky Way, leaving that discussion to Section 3.4. We first assume the simplest possible configuration: an isotropic sphere in which mass follows light. Further assuming that the density is described as a King model and is in virial equilibrium, Illingworth (1976) showed that the total mass is then $M=167 \beta r_{c} \sigma^{2}$ where $\beta$ is a parameter that depends on the concentration of the system and is generally assumed to be 8 for dSphs (Mateo 1998), $r_{c}$ is the King (1966) profile core radius, and $\sigma$ is the observed average velocity dispersion. We convert the measured half-light radius of Segue 1 to King core radius as $r_{c}=0.64 * r_{\text {eff }}=18.6_{-3}^{+5} \mathrm{pc}$. Using this method, we estimate the total mass of Segue 1 to be $4.5_{-2.5}^{+4.7} \times 10^{5} M_{\odot}$.

Our second method to calculate the mass loosens the constraint that mass-follows-light and uses the individual stellar 
Table 2

Keck/DEIMOS Velocity Measurements for Stars in Segue 1 Sample

\begin{tabular}{|c|c|c|c|c|c|c|c|}
\hline Name & $\begin{array}{c}\alpha(\mathrm{J} 2000) \\
\mathrm{h} \mathrm{m} \mathrm{s}\end{array}$ & $\begin{array}{c}\delta(\mathrm{J} 2000) \\
0, \quad "\end{array}$ & $\begin{array}{c}g \\
\mathrm{mag}\end{array}$ & $\begin{array}{c}(g-r) \\
\text { mag }\end{array}$ & $\begin{array}{c}v \\
\mathrm{~km} \mathrm{~s}^{-1} \\
\end{array}$ & $\begin{array}{c}\begin{array}{c}v_{\text {err }} \\
\mathrm{km} \mathrm{s}^{-1}\end{array} \\
\end{array}$ & $\begin{array}{c}v_{\mathrm{gsr}} \\
\mathrm{km} \mathrm{s}^{-1} \\
\end{array}$ \\
\hline \multicolumn{8}{|c|}{ Segue 1 Members } \\
\hline 3451635 & $10: 06: 40.5$ & $+16: 02: 38.1$ & 22.0 & 0.36 & 204.1 & 6.4 & 109.2 \\
\hline 3451345 & $10: 06: 44.5$ & $+16: 01: 29.4$ & 20.7 & 0.27 & 210.5 & 4.0 & 115.5 \\
\hline 3451159 & 10:06:44.6 & $+15: 59: 53.9$ & 17.3 & -0.01 & 200.4 & 2.2 & 105.5 \\
\hline 3451358 & $10: 06: 49.1$ & $+16: 03: 48.7$ & 20.6 & 0.22 & 198.9 & 5.1 & 104.0 \\
\hline 3451685 & $10: 06: 49.6$ & $+16: 03: 08.3$ & 21.1 & 0.13 & 207.8 & 6.7 & 112.9 \\
\hline 3451364 & $10: 06: 52.3$ & $+16: 02: 35.8$ & 18.9 & 0.48 & 215.6 & 2.9 & 120.7 \\
\hline 3451423 & 10:06:55.4 & $+16: 04: 16.2$ & 20.7 & 0.27 & 213.0 & 3.8 & 118.1 \\
\hline 3451533 & $10: 06: 57.4$ & $+16: 03: 00.0$ & 21.6 & 0.29 & 216.8 & 4.2 & 121.9 \\
\hline 3451726 & 10:06:57.6 & $+16: 02: 30.1$ & 22.3 & 0.14 & 212.8 & 5.3 & 117.9 \\
\hline 3451735 & $10: 06: 59.8$ & $+16: 02: 18.5$ & 22.0 & 0.38 & 203.6 & 4.9 & 108.7 \\
\hline 3451382 & 10:07:03.2 & $+16: 03: 35.0$ & 21.8 & 0.34 & 206.6 & 5.2 & 111.7 \\
\hline 3451378 & 10:07:03.3 & $+16: 02: 34.4$ & 20.6 & 0.29 & 205.5 & 2.7 & 110.6 \\
\hline 3451306 & 10:07:05.6 & $+16: 04: 22.0$ & 17.5 & -0.08 & 198.7 & 2.3 & 103.8 \\
\hline 3451374 & 10:07:01.3 & $+16: 02: 00.0$ & 20.5 & 0.25 & 208.7 & 2.7 & 113.8 \\
\hline 3451757 & $10: 07: 01.5$ & $+16: 03: 04.4$ & 22.4 & 0.12 & 200.4 & 6.1 & 105.5 \\
\hline 3451790 & $10: 07: 06.7$ & $+16: 04: 44.4$ & 21.8 & 0.29 & 206.5 & 6.7 & 111.6 \\
\hline 1894468 & 10:07:14.8 & $+16: 06: 27.1$ & 22.7 & 0.57 & 205.2 & 5.4 & 110.3 \\
\hline 3517005 & $10: 07: 14.9$ & $+16: 04: 48.8$ & 21.0 & 0.27 & 207.2 & 3.7 & 112.3 \\
\hline 1894643 & $10: 07: 15.1$ & $+16: 07: 08.2$ & 21.7 & 0.39 & 206.3 & 6.5 & 111.4 \\
\hline 3517002 & 10:07:15.7 & $+16: 03: 00.0$ & 21.2 & 0.14 & 206.4 & 13.1 & 111.5 \\
\hline 3517007 & $10: 07: 16.3$ & $+16: 03: 40.3$ & 21.7 & 0.26 & 198.4 & 4.4 & 103.5 \\
\hline 3516925 & $10: 07: 24.1$ & $+16: 04: 29.9$ & 22.1 & 0.32 & 197.1 & 7.6 & 102.2 \\
\hline 1894761 & $10: 07: 28.4$ & $+16: 07: 41.2$ & 22.4 & 0.56 & 216.9 & 14.3 & 122.0 \\
\hline 3517048 & $10: 07: 31.1$ & $+16: 04: 19.5$ & 21.6 & 0.32 & 212.4 & 9.8 & 117.5 \\
\hline \multicolumn{8}{|c|}{ Higher Velocity Stars } \\
\hline 3451696 & $10: 06: 50.8$ & $+16: 03: 51.2$ & 22.1 & 0.16 & 312.1 & 11.9 & 217.2 \\
\hline 3517146 & $10: 07: 13.7$ & $+16: 04: 44.8$ & 22.1 & 0.35 & 299.7 & 3.6 & 204.8 \\
\hline 3516836 & $10: 07: 17.4$ & $+16: 03: 55.6$ & 20.1 & 0.32 & 295.4 & 2.4 & 200.5 \\
\hline 3517243 & $10: 07: 32.5$ & $+16: 05: 00.5$ & 22.6 & 0.52 & 280.6 & 6.9 & 185.7 \\
\hline \multicolumn{8}{|c|}{ Non-Members } \\
\hline 3451324 & $10: 06: 35.5$ & $+16: 02: 21.1$ & 17.7 & 0.88 & 1.8 & 2.2 & -93.1 \\
\hline 3451835 & $10: 06: 36.3$ & $+16: 02: 46.3$ & 23.2 & 1.22 & -20.8 & 2.7 & -115.7 \\
\hline 3451597 & $10: 06: 34.8$ & $+15: 59: 48.8$ & 21.6 & 0.78 & -161.1 & 2.9 & -256.0 \\
\hline 3451847 & $10: 06: 37.8$ & $+16: 02: 22.6$ & 23.2 & 1.75 & 56.9 & 2.3 & -38.1 \\
\hline 3451828 & 10:06:37.9 & $+15: 59: 34.4$ & 23.1 & 1.02 & -25.4 & 3.0 & -120.3 \\
\hline 3451832 & $10: 06: 38.2$ & $+15: 59: 51.2$ & 23.0 & 0.57 & 32.7 & 8.1 & -62.2 \\
\hline 3451450 & $10: 06: 43.5$ & $+16: 02: 39.9$ & 22.6 & 1.67 & 14.4 & 2.3 & -80.5 \\
\hline 1829185 & $10: 06: 51.0$ & $+16: 04: 25.3$ & 21.8 & 0.44 & 97.6 & 3.2 & 2.7 \\
\hline 3451760 & $10: 07: 03.0$ & $+16: 01: 36.4$ & 21.8 & 0.35 & 155.9 & 5.9 & 61.0 \\
\hline 3451544 & 10:07:03.3 & $+16: 01: 40.6$ & 21.2 & 0.19 & 210.0 & 21.1 & 115.1 \\
\hline 3451424 & 10:06:58.5 & $+16: 00: 59.9$ & 20.7 & 1.28 & -14.3 & 2.2 & -109.2 \\
\hline 3516987 & $10: 07: 08.9$ & $+16: 04: 45.6$ & 21.8 & 0.29 & 205.9 & 23.1 & 111.0 \\
\hline 3451388 & $10: 07: 07.7$ & $+16: 02: 43.2$ & 18.5 & 0.53 & -34.4 & 2.2 & -129.3 \\
\hline 1894285 & $10: 07: 14.0$ & $+16: 06: 08.9$ & 18.0 & 0.31 & -174.2 & 2.2 & -269.1 \\
\hline 3517144 & $10: 07: 14.6$ & $+16: 03: 02.5$ & 22.5 & 0.92 & -117.1 & 2.5 & -212.1 \\
\hline 1894362 & $10: 07: 15.4$ & $+16: 07: 35.0$ & 22.9 & 1.26 & 17.2 & 2.2 & -77.7 \\
\hline 1894374 & 10:07:19.5 & $+16: 08: 04.9$ & 17.1 & 0.97 & 22.9 & 2.2 & -72.1 \\
\hline 3516717 & $10: 07: 20.5$ & $+16: 03: 16.1$ & 17.7 & 0.56 & -31.8 & 2.2 & -126.7 \\
\hline 3516719 & $10: 07: 21.1$ & $+16: 03: 34.5$ & 16.2 & 0.58 & 19.4 & 2.2 & -75.5 \\
\hline 3516859 & $10: 07: 25.1$ & $+16: 04: 45.4$ & 19.0 & 0.72 & 8.3 & 2.2 & -86.7 \\
\hline 3516748 & $10: 07: 26.2$ & $+16: 03: 54.3$ & 17.5 & 0.72 & -1.4 & 2.2 & -96.3 \\
\hline 3517219 & $10: 07: 28.9$ & $+16: 04: 46.7$ & 23.0 & -0.23 & 10.7 & 3.6 & -84.2 \\
\hline 1894306 & $10: 07: 34.3$ & $+16: 07: 00.9$ & 15.7 & 0.82 & -7.3 & 2.2 & -102.3 \\
\hline
\end{tabular}

Notes. Velocity measurements for member stars of Segue 1, higher velocity stars possibly associated with the Sgr stream and nonmembers. Positions and magnitudes are taken from the SDSS DR6. We list the heliocentric radial velocity $(v)$, velocity error $\left(v_{\text {err }}\right)$, and Galactocentric velocity $\left(v_{\mathrm{gsr}}\right)$ for each star as determined in Seciton 2.3 .

(This table is also available in a machine-readable form in the online journal.)

velocity measurements (in contrast to the velocity dispersion averaged over the projected radius as above). The method is described in Strigari et al. (2008a). Similar to the mass-follows- light method, this model assumes spherical symmetry and dynamical equilibrium, i.e., that the kinematic tracer population is related to the mass distribution via the Jeans equation. 

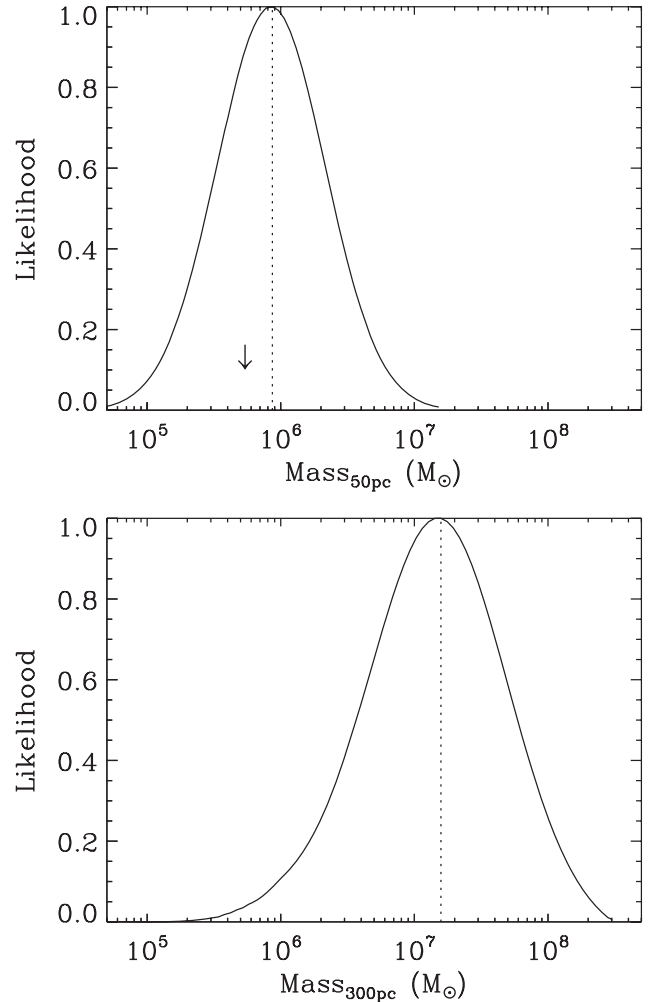

Figure 3. Likelihood distributions for the mass of Segue 1 enclosed within $50 \mathrm{pc}$ (top) and $300 \mathrm{pc}$ (bottom) determined using a two-component model as described in Section 3.3 and Strigari et al. (2008a). The arrow on the top plot indicates the mass of Segue 1 assuming mass follows light. The dotted lines show the best-fitting two-component model mass.

We assume that the light profile follows the observed Plummer profile with effective radius $r_{\text {eff }}=29 \mathrm{pc}$, and that the dark matter follows a five-parameter density profile characterized by a scale density, a scale radius, an asymptotic inner slope, an asymptotic outer slope, and a parameter governing the transition between these two slopes. The dark matter density profile allows for both flat central density cores and steep central density cusps, including the CDM-favored NFW-like $r^{-1}$ central cusps. We also allow for a radially varying stellar velocity anisotropy profile. We then marginalize over these five free parameters, and can estimate the mass at any given radius. Though the data do not constrain any of these parameters separately, the total dynamical mass within the stellar extent of $50 \mathrm{pc}$ is relatively well constrained. Using this model, we find the mass within $50 \mathrm{pc}$ to be $8.7_{-5.2}^{+13} \times 10^{5} M_{\odot}$. We note that the likelihood distribution of this quantity, shown in Figure 3, is nearly lognormal; the mass is greater than $5 \times 10^{4} M_{\odot}$ with $3 \sigma$ confidence. In comparison, the total stellar mass is merely $340 L_{\odot}$.

The two masses calculated above agree within errors. In the mass-follows-light method, the majority of the dark matter mass in the galaxy resides within the stellar radius, while the second method leaves open the possibility that the majority of the mass lies outside the observed light distribution. However, determining the total galaxy mass requires knowledge of the total radial extent of the dark matter halo and the profile shape beyond the last observed point. This is clearly difficult to determine observationally and strongly depends on the unknown orbital history of the galaxy. We can only estimate the instantaneous tidal radius of the galaxy, which ranges from a few ten to a few hundred parsecs depending on assumptions detailed in Section 3.4.2. If the tidal radius is large, then it is plausible that the majority of mass lies outside the stellar distribution.

Extrapolating the second estimate of mass to a radius of $300 \mathrm{pc}$, we find a total dynamical mass of $10^{7} M_{\odot}$, which, remarkably, is consistent with the common mass scale of all Milky Way dSphs (Strigari et al. 2008a). This common mass scale has been noted in previous studies (Mateo et al. 1993; Gilmore et al. 2007); we discuss this further in Section 6. If the stellar component of Segue 1 is embedded in a $10^{7} M_{\odot}$ dark matter halo, we would expect the luminous component to have experienced very little tidal disruption despite its current proximity to the Galaxy.

Regardless of which estimator is used above, the observed mass of Segue 1 is significantly larger than expected if all its mass were due to a stellar-only component. Since Segue 1 contains little to no HI gas (Putman et al. 2008), the stellar mass likely dominates the total baryonic mass. In the absence of nonbaryonic dark matter, we expect the M/L of Segue 1 to be $\mathrm{M} / \mathrm{L}_{V} \sim 3$, accounting for stellar remnants in an old stellar population (Maraston 2005). Assuming this $\mathrm{M} / \mathrm{L}$, the stellar mass of Segue 1 is $\sim 1 \times 10^{3} M_{\odot}$, translating into an expected velocity dispersion of merely $0.4 \mathrm{~km} \mathrm{~s}^{-1}$. This is more than $3 \sigma$ below the measured dispersion of Segue 1 and thus argues strongly for the presence of dark matter.

Finally, we calculate the $v$-band $\mathrm{M} / \mathrm{L}\left(\mathrm{M} / \mathrm{L}_{V}\right)$ within the observed radius. Combining the absolute luminosity of Segue 1 $\left(M_{V}=-1.5_{-0.8}^{+0.6}\right)$ with the mass from the first method above (assuming mass-follows-light), we calculate a $\mathrm{M} / \mathrm{L}$ of $\ln \left(M / L_{V}\right)=7.2_{-1.2}^{+1.1}\left(\mathrm{M} / \mathrm{L}_{V}=1320_{-936}^{+2684}\right)$, and in the second two-component method we calculate $\ln \left(M / L_{V}\right)=7.8_{-1.3}^{+0.5}$ $\left(\mathrm{M} / \mathrm{L}_{V}=2440_{-1775}^{+1580}\right)$. In both cases, the error distribution is asymmetric and the $\mathrm{M} / \mathrm{L}$ is well in excess of that predicted from the stellar mass alone. The two-component model ratios suggests a dark matter-dominated galaxy with a $6 \sigma$ significance. If the luminous components of dSphs do indeed reside in common mass dark matter halos, we would predict the highest $\mathrm{M} / \mathrm{Ls}$ in the least luminous dSphs (see the middle panel of Figure 6). Since Segue 1 is the least luminous of the recently discovered ultra-faint Milky Way satellites, this remarkably high $\mathrm{M} / \mathrm{L}$ is expected in this model. Understanding the processes that lead to this high $\mathrm{M} / \mathrm{L}$ will be a future challenge to galaxy formation models.

\subsection{Possible Caveats on the Mass of Segue 1}

The remarkably high $\mathrm{M} / \mathrm{L}$ of Segue 1 rests on our interpretation that the measured stellar velocities faithfully trace the gravitational potential. Here we discuss two possibilities that might affect this assumption. First is the presence of unresolved binary stars inflating our measured velocity dispersion. The second is tidal interactions with the Milky Way affecting the kinematics. Both issues are difficult to quantify without further observations, but are worthwhile considering here.

\subsubsection{The Effects of Binary Stars}

If a high percentage of our Segue 1 stellar members are in fact unresolved binary star systems, the measured velocity dispersion may be inflated due to their orbital motion. The severity of this effect depends on the mass ratio of individual systems, with equal mass binaries contributing most to the velocity bias. The likely presence of binaries in our kinematics sample is difficult to estimate empirically without repeated velocity measurements. Olszewski et al. (1996) simulate the 
effects of binaries on the velocity dispersions of dSph with data sets somewhat similar to that of Segue 1. Assuming a solar neighborhood binary fraction, they suggest that the velocity dispersion due to binaries alone is on the order $\sim 1.5 \mathrm{~km} \mathrm{~s}^{-1}$. Thus, while the overall dispersion may be inflated by binaries, they cannot explain away the strong evidence of dark matter. Because the true binary fraction in Segue 1 may be very different from that of the solar neighborhood, we do not fold this systematic error into our mass estimates. Additional observations and improved simulations will allow us to better quantify the effects of binaries on our results.

\subsubsection{The Effects of Tidal Interactions}

It is likely that Segue 1 has been affected by tidal interactions with the Galaxy. Segue 1 lies at a distance of $23 \mathrm{kpc}$ from the Sun, or $\mathrm{d}_{\mathrm{GC}-\mathrm{Seg} 1}=28 \mathrm{kpc}$ from the Galactic Center (GC) assuming a GC-Sun distance of $8.5 \mathrm{kpc}$. We estimate the instantaneous tidal radius for Segue 1 by first approximating both the Milky Way and Segue 1 as point masses. We assume a enclosed Milky Way mass of $3 \times 10^{11} M_{\odot}$, corresponding to a circular velocity of $210 \mathrm{~km} \mathrm{~s}^{-1}$ at $28 \mathrm{kpc}$. This is consistent with the Milky Way model used in Section 4 and Law et al. (2005). Using the first estimate for the mass of Segue 1 in Section 3.3, we determine a tidal radius of $r_{\mathrm{t}}=\left(M_{\mathrm{Seg} 1} / M_{\mathrm{MW}}\right)^{(1 / 2)} d_{\mathrm{GC}-\mathrm{Seg} 1}=$ 33 pc. Assuming that Segue 1 is embedded in an extended dark matter halo (using mass from the second method above at $300 \mathrm{pc}$ ), the tidal radius increases to $r_{\mathrm{t}}=160 \mathrm{pc}$. In the first case, the luminous matter extends beyond the tidal radius and we would expect to see evidence for unbound stars in our kinematic sample, in the second case we would expect our observations to be well within the bound radius. However, this calculation does not account for the fact that Segue 1 orbits the Milky Way. When calculating the tidal radius in the reference frame of the MW-Segue 1 system, stars in Segue 1 will also feel centrifugal and coriolis forces in this rotating reference frame. If we include these forces, the tidal radius (also called the Jacobi or Roche radius, Binney \& Tremaine (2008), Equation 8.91) is then $r_{\mathrm{t}}=\left(M_{\mathrm{Seg} 1} / 3 * M_{\mathrm{MW}}\right)^{1 / 3} d_{\mathrm{GC}-\mathrm{Seg} 1}$. The tidal radius then increases to 220 and $615 \mathrm{pc}$ for the first and second cases, respectively. These are of course estimates of the instantaneous tidal radius: if Segue 1 is on an elliptical orbit the tidal radius may have been much different in the past.

If some, or all, of the stars associated with Segue 1 are tidally disrupting (unbound), then the measured velocities likely provide an inflated estimate of the mass (Klimentowski et al. 2007). In the extreme case that Segue 1 is completely unbound, its mass could be as low as the stellar component alone $\left(10^{3} M_{\odot}\right)$. However, the fact that the luminosity profile of Segue 1 is centrally concentrated suggests that this object is not completely unbound. The crossing time of Segue 1 (assuming $r_{\text {eff }}=30 \mathrm{pc}$ and a velocity dispersion of $4.3 \mathrm{~km} \mathrm{~s}^{-1}$ ) is $10^{7}$ years. The travel time along the orbit of Segue 1 in $10^{7}$ years is roughly $2 \mathrm{kpc}$ assuming a circular orbital speed of $200 \mathrm{~km} \mathrm{~s}^{-1}$. Thus, we would naively expect an unbound version of Segue 1 reside only a few kpc away from its disruption site before quickly dissipating, making this extreme scenario unlikely. Detailed dynamical modeling is appropriate to this system and a knowledge of Segue 1's orbital history is required to properly determine the degree of tidal interactions and disruption in this system. Our mass estimates presented in this paper explicitly assume that the kinematics of Segue 1 are not affected by tides.

To determine the true orbit of Segue 1, we need to know its transverse motion. The heliocentric velocity of Segue 1 is $v=206 \mathrm{~km} \mathrm{~s}^{-1}$ and the velocity relative to the Galactic Standard of $\operatorname{Rest}^{9}$ (GSR) is $v_{\mathrm{GSR}}=114 \mathrm{~km} \mathrm{~s}^{-1}$. We can rule out a circular orbit: in the Milky Way model discussed in Section 4, the maximum projected GSR velocity for a circular orbit is $v_{\mathrm{GSR}, \text { circ }}=55 \mathrm{~km} \mathrm{~s}^{-1}$ at the distance of Segue 1. If the transverse motion of Segue 1 is similar to or less than that of the measured radial motion, then Segue 1 was closer to the Galactic Plane in the recent past. However, there is no clear evidence to suggest tidal stripping is currently affecting the luminous component. We do not see velocity outliers in our kinematic sample (which might indicate that these stars are in the process of being stripped), nor other clear evidence of on-going tidal disruption (e.g., photometric evidence of tidal tails or tidally induced rotation). While the absence of these features cannot be used as proof that tidal stripping is not on-going (Muñoz et al. 2008), it is consistent with our assumption that tidal stripping is not currently affecting the luminous component. An estimate of the proper motion of Segue 1 is needed to constrain the orbital history of this object.

\subsection{Metallicity}

We estimate the spectroscopic metallicity of individual stars in our Segue 1 sample via spectral synthesis modeling (Kirby et al. 2008a). The method compares the observed spectrum to a grid of synthetic spectra covering a range of effective temperature, surface gravity, and composition. We estimate effective temperature and surface gravity for each star based on the Johnson-Cousins VI magnitude which we determine by transforming the SDSS gri magnitudes (Chonis \& Gaskell 2008). The results are unaffected by using alternative photometric methods to determine these parameters. The best-matching composition is found by minimizing residuals between the observed spectrum and a smoothed synthetic spectrum matched to the DEIMOS spectral resolution. Our method has been tested against high-resolution Keck/HIRES abundances for six RGB stars in the ultra-faint dSphs of SG07 (Kirby et al. 2008b). This comparison yields precisions better than 0.25 dex for DEIMOS spectra with signal-to-noise ratios ( $\mathrm{S} / \mathrm{Ns}$ ) greater than $\mathrm{S} / \mathrm{N}>20 \AA^{-1}$. Although the method can theoretically be applied to all types of stars, it has not yet been tested against highresolution spectroscopic abundances for horizontal-branch or main-sequence stars.

Our kinematic sample contains a single RGB star $(r=18.4)$. The above method estimates its metallicity to be $[\mathrm{Fe} / \mathrm{H}]=$ $-3.3 \pm 0.2 \mathrm{dex}$. The effective temperature and surface gravity used to determine the metallicity of this star are $T_{\text {eff }}=5191 \mathrm{~K}$ and $\log g=2.76$, with estimated systematic errors of $150 \mathrm{~K}$ and 0.12 dex, respectively. The derived metallicity is much more sensitive to $T_{\text {eff }}$ than $\log g$; Kirby et al. (2008a) estimate that a $150 \mathrm{~K}$ change in $T_{\text {eff }}$ incurs an error on $[\mathrm{Fe} / \mathrm{H}]$ of less than 0.15 dex. While DEIMOS spectra contain some information about $\alpha$-element abundances, the errors we estimate on this quantity are large. The $[\mathrm{Fe} / \mathrm{H}]$ value is unchanged whether or not we mask out absorption lines due to the $\alpha$-elements.

A small portion of the observed Segue 1 RGB star spectrum and synthetic spectra are shown in Figure 4. At this metallicity, the strong absorption lines of $\mathrm{Ca}$ II are clearly visible, but weaker Fe lines are not. In the inset to Figure 4, we compare a small region of the observed spectrum to models at $[\mathrm{Fe} / \mathrm{H}]=-3.3$

\footnotetext{
9 To compute velocities in the Galactic standard of rest (GSR) frame, we assume the solar peculiar velocity is $(U, V, W)=(9,12,7) \mathrm{km} \mathrm{s}^{-1}$ relative to the local standard of rest, for which we adopt a rotation velocity of $220 \mathrm{~km} \mathrm{~s}^{-1}$.
} 


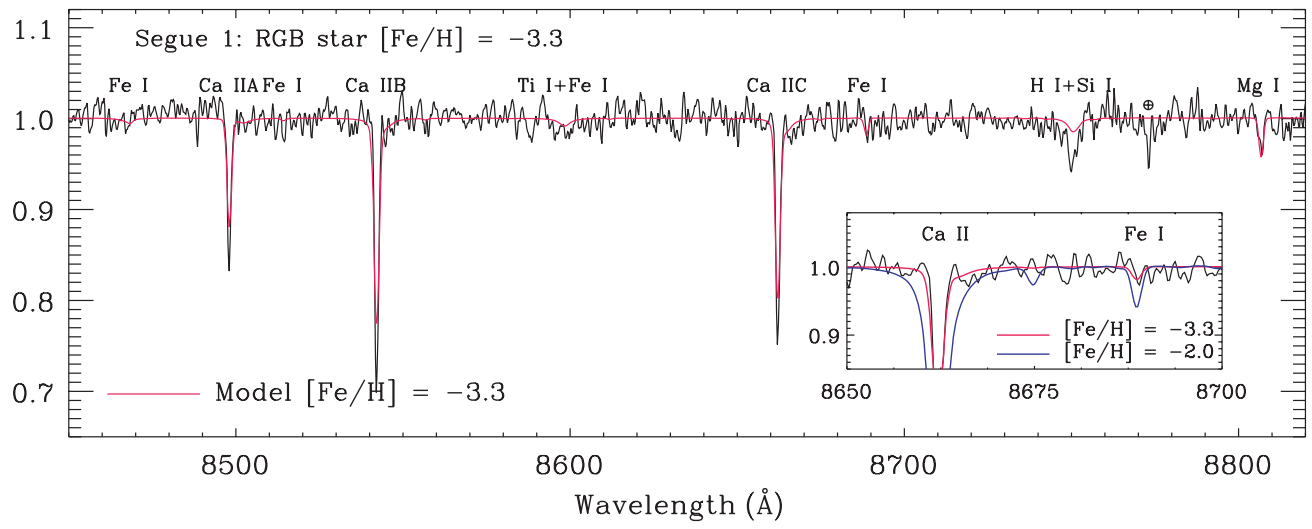

Figure 4. Keck/DEIMOS spectrum for the RGB star member of Segue 1. This star has a measured metallicity of $[\mathrm{Fe} / \mathrm{H}]=-3.3 \pm 0.2$ dex as determined via spectral synthesis (Kirby et al. 2008a). The model spectrum is shown in red. The cores of the Ca II triplet lines are not well modeled because they form out of local thermodynamic equilibrium and do not play any role in the metallicity determination. Inset is a zoom-in of a region near the Ca II $\mathrm{C}$-line. The red [Fe/ $\mathrm{H}] \mathrm{model}$ is compared to a more metal-rich model $([\mathrm{Fe} / \mathrm{H}]=-2.0$, blue line) with the same atmospheric parameters. The observed spectrum shows no evidence for absorption at $8689 \AA$, even though a star as metal poor as $[\mathrm{Fe} / \mathrm{H}]=-2.0$ would display this line.

(A color version of this figure is available in the online journal.)

and -2.0 with the same temperature and surface gravity. For a star with these parameters, the strongest Fe line in the DEIMOS spectral range is Fe I 8689. The observed spectrum in Figure 4 shows no evidence for absorption at $8689 \AA$, even though a more metal-rich star would display this line.

At red wavelengths, metallicity is often estimated via the Ca II triplet absorption lines (e.g., Helmi et al. 2006, SG07). However, Kirby et al. (2008b), Koch et al. (2008) and others note that current implementations of this method fail for metallicities below $[\mathrm{Fe} / \mathrm{H}] \sim-2.5$. The Ca II triplet method is based an empirical calibration of Galactic globular clusters and is not calibrated for metallicities below $[\mathrm{Fe} / \mathrm{H}] \leqslant-2.4$ (Rutledge et al. 1997). The metallicities in the ultra-faint $\mathrm{dSph}$ are below this limit. We therefore do not use this method and strongly caution the use of this relationship for very low metallicity systems. The remaining analysis is based on the results from the spectral synthesis method above.

While other stars in our kinematic sample have sufficient signal-to-noise to measure metallicity, $[\mathrm{Fe} / \mathrm{H}]$ estimates for the remainder of the sample are less reliable. The two horizontalbranch stars seen in Figure 1 are too hot to display strong metal absorption and what metal lines exist are overwhelmed by the Paschen series. The main-sequence stellar spectra are more suitable for metallicity measurement, but have much lower S/Ns as compared to the RGB star above and higher surface gravities. The synthesis method has also not yet been tested for stars with $\log g>3.3$. The main-sequence stars with adequate $\mathrm{S} / \mathrm{N}$ to measure a metallicity have surface gravities $3.5<\log g<4.3$. The average metallicity for these 13 main-sequence stars is $[\mathrm{Fe} / \mathrm{H}]=-1.8 \pm 0.1 \mathrm{dex}$, with individual measurements ranging from -1.5 to -2.8 . This average is significantly more metal rich than above and suggests that the mean metallicity of Segue 1 may be higher than that of the single RGB star. These results also suggest that Segue 1 has a significant internal metallicity spread. In support of this spread, we note that the fiducial isochrones in the color-magnitude diagram of Figure 1 cannot simultaneously fit the RGB and main sequence. While the horizontal-branch and main-sequence turnoff are well fitted in this figure, the single RGB star is slightly too blue, suggesting it is more metal poor than the main sequence, consistent with our spectroscopically measured metallicity. These results, however, should be approached with caution. While there are no obvious reasons the main-sequence metallicities should be biased, we remain aware that the spectral synthesis code has not been tested in this regime. Pending more reliable confirmation, we take the metallicity of the RGB star to be representative of Segue 1.

Kirby et al. (2008b) demonstrate that the luminositymetallicity relationship is loglinear for Milky Way dwarf galaxies across nearly four decades in luminosities (see Figure 6 and Section 6). Given the luminosity of Segue $1\left(M_{V}=-1.5, L_{V}=\right.$ $\left.340 L_{\odot}\right)$, the predicted metallicity based on this relationship is $[\mathrm{Fe} / \mathrm{H}]=-2.8 \pm 0.2$. While our metallicity estimate of the single RGB star in our Segue 1 sample is more metal poor than this prediction, the main-sequence metallicity is more metal rich. The average of these two metallicities is closer to the predicted value. Additional observations are required to securely determine whether or not Segue 1 lies on the luminosity-metallicity relationship, and in Figure 6 we assume that the average metallicity is equal to that of the RGB star. Quantifying the mean metallicity of Segue 1 and the amount of internal metallicity spread is crucial to interpreting the formation history of Segue 1. If this object does indeed lie on the luminosity-metallicity relationship and has a significant internal metallicity spread, this is further evidence for that Segue 1 formed via galaxy, rather than globular cluster, formation processes.

\section{SEGUE 1, DISTINCT FROM THE SAGITTARIUS STREAM}

Segue 1 is spatially super-imposed on the leading arm of the Sagittarius (Sgr) stream. This placement and its tiny size led Belokurov et al. (2007) to identify it as a possible globular cluster formerly associated with the Sgr dSph. Six other globular clusters have been associated with the Sgr stream (Bellazzini et al. 2003; Casetti-Dinescu et al. 2007; Carraro et al. 2007). While our measured velocity dispersion and inferred $\mathrm{M} / \mathrm{L}$ of Segue 1 suggest that it is not a globular cluster, it is still possible that it could have been a dwarf satellite of Sgr that has been captured by the Milky Way. We now investigate whether or not Segue 1 could be kinematically associated with the Sgr Stream.

Majewski et al. (2003) defined a longitudinal coordinate system, $\Lambda_{\odot}$, in which the center of the Sgr dSph lies at $\Lambda_{\odot}=0$. In this system, Segue 1 is roughly $130^{\circ}$ away from the main body of the Sgr dSph at $\Lambda_{\odot}=224.5$ (Figure 5). Unlike the region near the Sgr $\mathrm{dSph}$ or the trailing stream of recent tidal 

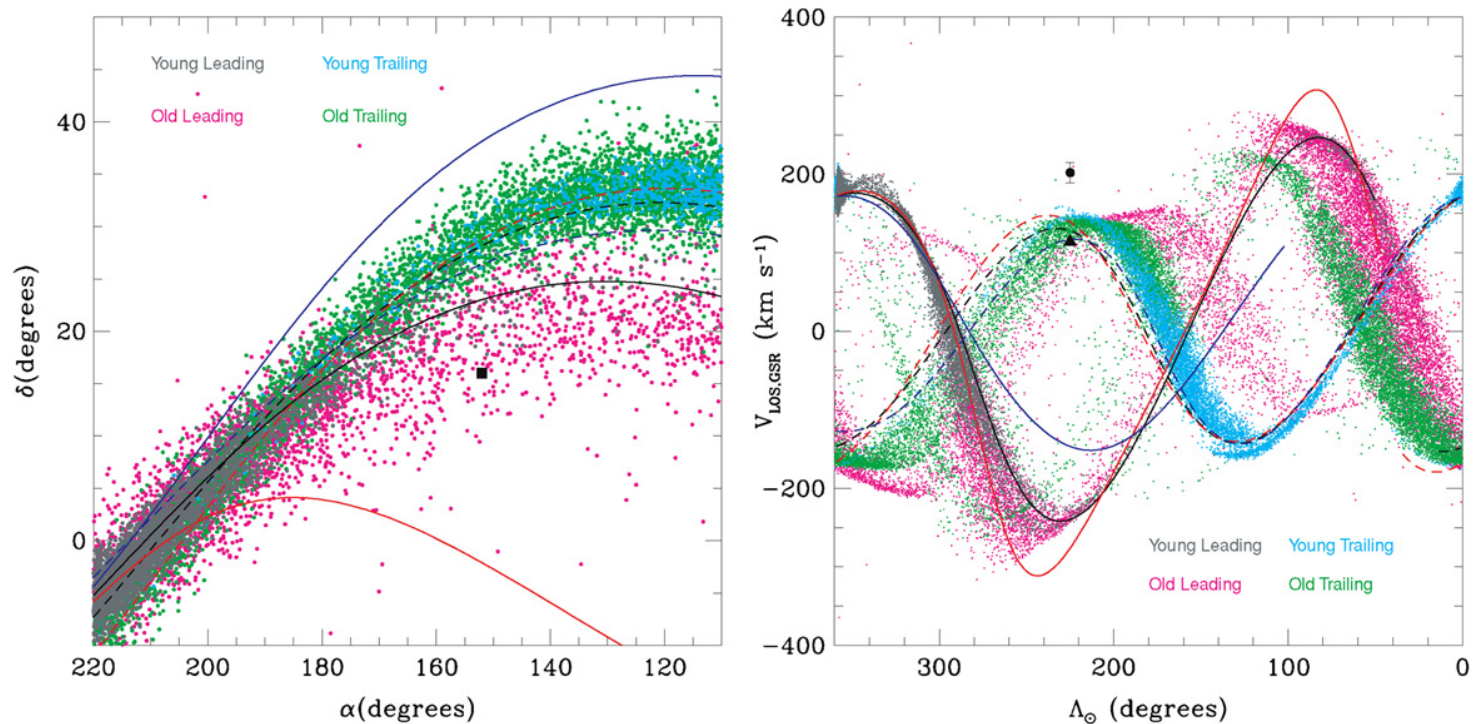

Figure 5. $N$-body model debris (colored points) from the Sgr dSph is plotted as a function of (left) sky coordinates and (right) line-of-sight (LOS) velocity (relative to the GSR) as a function of orbital longitude $\Lambda_{\odot}$ (Majewski et al. 2003). Gray/cyan points represent debris lost from Sgr during the last 5 Gyr and magenta/green points are debris lost more than $5 \mathrm{Gyr}$ ago in the leading/trailing tidal tails, respectively. The orbit of the Sgr dwarf core (black line) shown as a solid/dashed line for leading/trailing portions of the orbit, respectively, is overplotted. The red/blue lines respectively represent the orbits of the $q=0.9$ and 1.25 and models of Law et al. (2005) for comparison. The location of the Segue 1 field is indicated by a square in the left panel, and the velocity of the Segue I dwarf (triangle) and high-velocity feature (circle) are indicated in the right-hand panel. The error bars on the high-velocity feature indicate the $1 \sigma$ spread about the mean value of stars in the feature. (A color version of this figure is available in the online journal.)

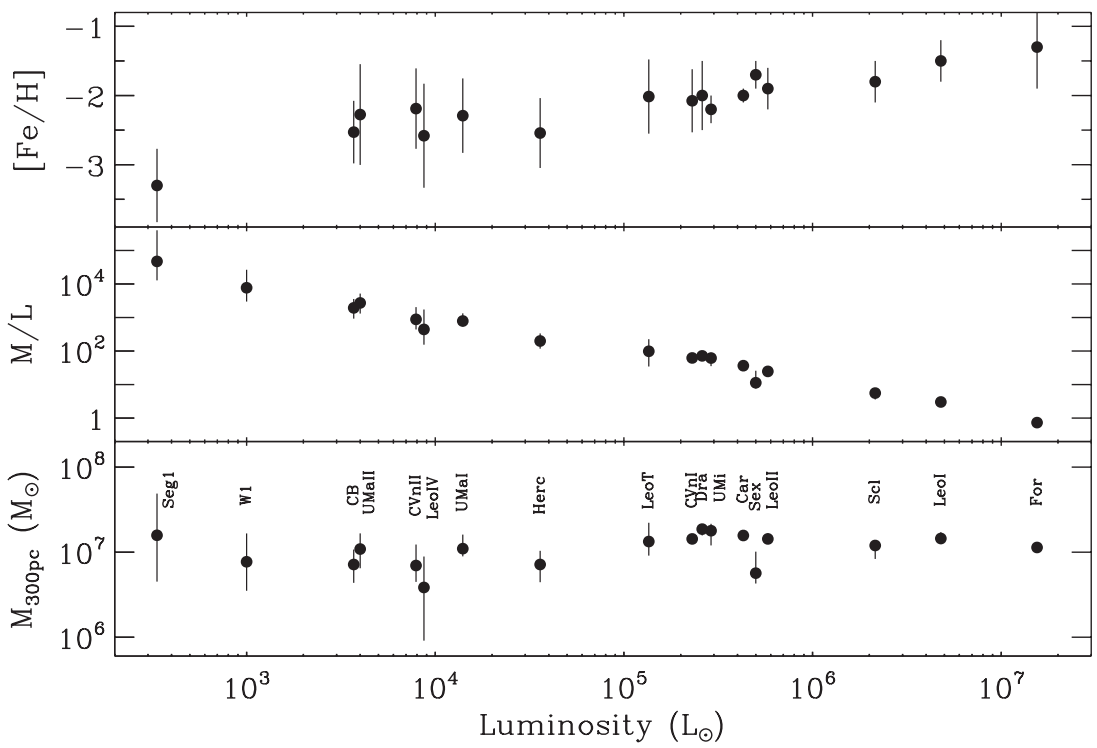

Figure 6. Segue 1 lies at the low-luminosity end of the metallicity $([\mathrm{Fe} / \mathrm{H}])$-luminosity, mass-to-light-luminosity and mass-luminosity relationships established by the Milky Way dSphs. Masses for the Milky Way dSph are taken from Strigari et al. (2008a), luminosities from Martin et al. (2008) and Mateo (1998), and metallicities from Kirby et al. (2008b). While the luminosity spans nearly five orders of magnitude, the enclosed (300 pc) remains nearly constant at $10^{7} M_{\odot}$. Over the same luminosity range, the metallicity decreases nearly 2 dex. Explaining the mechanisms that set these relationships is key to understanding galaxy formation at the smallest scales.

debris $\left(\Lambda_{\odot} \sim 0^{\circ}-100^{\circ}\right)$, the kinematics of the stream in the region near Segue 1 are not well determined observationally. We therefore compare our data to numerical $N$-body models in order to determine whether Segue may be kinematically as well as spatially associated with Sgr tidal debris.

Our model of the Sgr stream is similar in many respects to the $q=1.0$ model (i.e., that in which the Galactic dark halo potential is spherical) described by Law et al. (2005), with some modifications made in order to simultaneously match both the trailing arm M-giant velocities (Majewski et al. 2004) and the newly observed SDSS leading arm bifurcation (Belokurov et al. $2006 \mathrm{~b})$. In brief, the Galactic halo flattening in this model is mildly prolate $(q=1.05)$, and the model Sgr dwarf has an initial mass of $10^{8} M_{\odot}$, a scale length of $350 \mathrm{pc}$, and has been orbiting in a static Galactic potential for $\sim 9$ Gyr. We refer the reader to Law et al. (2005) for a more thorough discussion of the $N$-body modeling technique.

Figure 5 (left panel) illustrates the previously noted spatial alignment of the Segue 1 field with the leading Sgr stream (i.e., the "A" and "C" streams of Fellhauer et al. 2006). As demonstrated in the right hand panel, however, the velocity relative to the GSR of Segue $1\left(v_{\mathrm{Seg} 1, \mathrm{GSR}}=114 \mathrm{~km} \mathrm{~s}^{-1}\right)$ is wildly discrepant with the leading tidal stream at the corresponding angular position $\left(\sim-250 \mathrm{~km} \mathrm{~s}^{-1}\right.$, gray/magenta points). 
Instead, Segue 1 appears to be more consistent in velocity with the trailing stream, from which it is offset by $\sim 15^{\circ}(\sim 6 \mathrm{kpc}$ at the distance of Segue 1). Given these conflicting data, we conclude that Segue 1 is not physically associated with either stream.

We note for completeness however that Segue 1 is consistent in both angular coordinates and radial velocity with an extremely old segment of leading arm tidal debris (released from $\mathrm{Sgr} \sim$ $7-8$ Gyr ago) which has been wrapped roughly $520^{\circ}$ around the Milky Way from the Sgr core (i.e., the segment of magenta debris at $\Lambda_{\odot} \sim 220^{\circ}$ and $V_{\mathrm{LOS}, \mathrm{GSR}} \sim 100 \mathrm{~km} \mathrm{~s}^{-1}$ ). However, conclusive observational evidence for the existence of such old, multiply-wrapped tidal debris from Sgr has not yet been established. Our models therefore leave open the possibility that Segue 1 was initially associated with the Sgr dSph, but was removed very early in the tidal interactions between Sgr and the Milky Way. Previous claims of associated cluster systems (e.g., Bellazzini et al. 2003; Casetti-Dinescu et al. 2007) have focused only on relatively recent debris.

There is of course still considerable uncertainty in the "best" model for Sgr. Only models with strongly prolate $(q=1.25)$ halos produce streams that match the leading arm M-giant velocities Law et al. (2005), while models with oblate halos ( $q=$ 0.9 ) best match the observed precession of the M-giant orbital plane (Johnston et al. 2005). In contrast, a near spherical model is required in order to match the bifurcated stream observed in the SDSS (Belokurov et al. 2006b), as is an extremely low satellite mass $\left(10^{8} M_{\odot}\right)$ which in turn produces streams too dynamically cold to reproduce the observed dispersion among the M-giant velocities (Law et al. 2005). In Figure 5, we demonstrate the behavior of Sgr debris in oblate, near-spherical, and prolate Galactic dark halo potentials via point-particle orbital tracks (red, black, and blue lines, respectively). While debris from an $\mathrm{N}$-body satellite is not perfectly traced by the orbit of the satellite core, this orbital track gives a good sense of the behavior of the debris (note how the black line roughly tracks the colored points) and indicates that it is not possible to construct a model in which leading tidal debris can match the observed velocity of Segue 1. Similarly, for no model are trailing Sgr debris spatially coincident with Segue 1 while simultaneously reproducing the trend of trailing M-giant velocities observed by Majewski et al. (2004). While puzzles obviously remain, these uncertainties do not affect our conclusion that Segue 1 cannot be associated with recent Sgr debris.

\subsection{Higher Velocity Stars: An Old Piece of the Sagittarius Stream?}

There are four stars in our kinematic sample with $v \sim$ $300 \mathrm{~km} \mathrm{~s}^{-1}$, or $v_{\mathrm{GSR}} \sim 200 \mathrm{~km} \mathrm{~s}^{-1}$ (Figure 2). This is unusual in that standard Milky Way models predict that such high-velocity stars are extremely rare (e.g., Robin et al. 2003). These four stars have sufficiently different velocities $\left(\Delta v=30 \mathrm{~km} \mathrm{~s}^{-1}\right)$ that they are not gravitationally bound to each other; however, given their spatial and kinematic proximity they could plausibly be associated with a single stellar stream. To highlight how unusual this grouping is, we note that over the eight fields observed by SG07 (with similar targeting priorities), only seven out of nearly 900 stars had $v_{\mathrm{GSR}} \geqslant 200 \mathrm{~km} \mathrm{~s}^{-1}$, with only one field having more than one higher velocity stars (as compared to four of 59 in Segue 1). Since none of the SG07 fields are near any known streams, we circumstantially associated these four stars with the Sgr stream. However, none of the Sgr models discussed above match the position and velocity of these higher velocity stars (filled circle in Figure 5). We tentatively associate these stars with older Sgr tidal debris or a possibly new stream. More observations and theoretical work are needed in this region to confirm this hypothesis.

\section{THE PREDICTED GAMMA-RAY FLUX FROM SEGUE 1}

Having established that Segue 1 is dark matter-dominated, it is interesting to consider the implications of having a massive dark matter halo in such close proximity to the Sun. Generically, dSphs have been attractive targets for indirect dark matter detection experiments, via particle annihilation production of gamma rays, due to their high dark matter densities and lack of internal gamma-ray sources (Baltz et al. 2000; Tyler 2002). Strigari et al. (2008b) note that the even higher dark matter densities of the recently discovered ultra-faint dSphs, combined with their proximity, make them particularly interesting candidates for indirect detection. Upper limits on the gamma-ray fluxes have so far been reported for several classical dwarfs including the Draco, Ursa Minor, and Sagittarius dSph (Aharonian et al. 2008; STACEE Collaboration: Driscoll et al. 2007; Wood et al. 2008). The recent launch of Gamma-ray Large Area Telescope (GLAST) satellite (Ritz et al. 2007) makes this a particularly timely calculation.

Based on the mass estimates of Section 3.3, the average dark matter density of Segue 1 is $1.65 M_{\odot} / \mathrm{pc}^{-3}$ inside $50 \mathrm{pc}$. We determine the gamma-ray flux from dark matter annihilation by marginalizing over the unknown halo parameters using a maximum-likelihood analysis similar to that described in Section 3.3. We assume the most optimistic supersymmetric model for the dark matter particle, and refer to Strigari et al. (2008b) for additional details of the input assumptions. Because we are considering an annihilation signal, the gamma-ray flux scales as the square of the central density and as the inverse square of the distance. Marginalizing over the appropriate parameters, we find the predicted gamma-ray flux for Segue 1 is $\Phi_{\gamma}=5.5_{-3.5}^{+10} \times 10^{-10}$ photons $\mathrm{cm}^{-2} \mathrm{~s}^{-1}$. The mean value of this flux is more than a factor of 10 larger than that from the classical dSphs and is higher than that predicted for any of the previous ultra-faint dwarfs (Strigari et al. 2008b). Thus, Segue 1 is an excellent target for the indirect detection of dark matter via particle annihilation.

\section{DISCUSSION}

As seen in Figure 6, Segue 1 lies on an extension of the luminosity-metallicity and luminosity-mass relationships established by brighter Milky Way dSphs. While the dSphs span nearly five orders of magnitude in luminosity, their mass enclosed within $300 \mathrm{pc}$ remains nearly constant at $10^{7} M_{\odot}$ (Strigari et al. 2008a). This common mass scale has been noted in previous studies (Mateo et al. 1993; Gilmore et al. 2007), but remains a very surprising result given the much larger luminosity range spanned by the present data. It strongly suggests the existence of a characteristic scale in either galaxy formation processes or dark matter physics. At the same time, the average metallicities of the dSphs are correlated with luminosity such that stars in the least-luminous dSph are the most metal poor (Kirby et al. $2008 \mathrm{~b}$ ). Segue 1 is at the extreme end of these relationships: its luminosity is merely $L=340 L_{\odot}$, yet its total mass enclosed within $300 \mathrm{pc}$ is $10^{7} M_{\odot}$ (projecting the mass model discussed in Section 3.3), resulting in the highest $\mathrm{M} / \mathrm{L}_{V}$ of any known stellar system. The metallicity for the single RGB star in Segue 1 is $[\mathrm{Fe} /$ $\mathrm{H}]=-3.3 \mathrm{dex}$, one of the most metal-poor stars known in a dSph 
galaxy. This metallicity is slightly less than that predicted by the Kirby et al. loglinear relationship; however, we note that the average galactic metallicity may be higher than this single star.

The correlations in Figure 6 are the key to understanding how $\mathrm{dSphs}$ form. While several formation avenues exist to modify the $\mathrm{M} / \mathrm{L}$ of $\mathrm{dSphs}$, the added constraint of the luminositymetallicity correlation reduces the number of allowable models. This correlation rules out a tidal stripping scenario in which lower luminosity systems initially form as more luminous galaxies outside the environment of the Milky Way and are then tidally stripped to their present state as they enter the Milky Way environs. In this scenario, the metallicity of stars would not be tied to the present luminosity. While ruling out formation scenarios is certainly progress, determining what formation processes can explain the observed correlations will be more challenging (e.g., Bovill \& Ricotti 2008). A key question raised by the Segue 1 results is why the Milky Way dwarf dSphs have such remarkably different luminosities, yet appear to have similar total masses. Why do all these objects have a common mass halo and is this consistent with the mass spectrum of dark matter halos predicted by simulations? Explaining the mechanism that sets both the mass-luminosity and luminositymetallicity relationships in the Milky Way will provide insight into the formation of galaxies at all scales.

\section{SUMMARY}

Segue $1\left(M_{V}=-1.5_{-0.8}^{+0.6}\right)$ is the least luminous of the ultra-faint galaxies recently discovered in the SDSS, and thus the least luminous known galaxy. We present Keck/DEIMOS spectroscopy of 24 member stars that suggests that Segue 1 is dark matter-dominated and metal poor. We measure an internal velocity dispersion of $4.3 \pm 1.2 \mathrm{~km} \mathrm{~s}^{-1}$, and infer a total mass of $4.5_{-2.5}^{+4.7} \times 10^{5} M_{\odot}$ in the case where mass-follow-light; using a two-component maximum-likelihood model, we determine a mass within $50 \mathrm{pc}$ of $8.7_{-5,3}^{+13} \times 10^{5} M_{\odot}$. The two masses agree within errors, however, in the first case, the majority of dark matter mass in the galaxy resides within the stellar radius, while the second method leaves open the possibility that the majority of the mass lies outside the observed light distribution. The metallicity of the single RGB star in our sample is $[\mathrm{Fe} / \mathrm{H}]$ $=-3.3$ dex, well below that of any known globular cluster Harris (1996). Although Segue 1 is spatially superimposed on the Sagittarius stream, its mean velocity is inconsistent with recent Sagittarius tidal debris in this region. Our models leave open the possibility that Segue 1 is a dwarf galaxy that was initially associated with the Sgr $\mathrm{dSph}$, but was stripped away early in tidal interaction between Sagittarius and the Milky Way. Finally, we note that the combined high central dark matter density and the proximity of Segue 1 make it an attractive target for indirect dark matter detection experiments.

The number of ultra-faint dSphs around the Milky Way has doubled in the past few years. The seemingly ubiquitous presence of these objects has forced a fundamental shift in galaxy formation models at the smallest scales. The high $\mathrm{M} / \mathrm{Ls}$ and dark matter densities of the least luminous dSphs, such as Segue 1, may also lead to an improved understanding of dark matter itself. The promised discovery of many additional ultra-faint dwarfs around the Milky Way and other nearby galaxies makes this fertile ground for continued study.

We acknowledge and appreciate conversations regarding this work with James Bullock, Raja Guhathakurta, Manoj Kaplinghat, Shane Walsh, and Adi Zolotov. E.N.K. acknowledges the support of an NSF Graduate Research Fellowship. J.D.S. gratefully acknowledges the support of a Millikan Fellowship provided by Caltech. L.E.S. by NASA through Hubble Fellowship grant HF-01225.01 awarded by the Space Telescope Science Institute, which is operated by the Association of Universities for Research in Astronomy, Inc., for NASA, under contract NAS $5-26555$.

\section{REFERENCES}

Adelman-McCarthy, J. K., et al. 2008, ApJS, 175, 297

Aharonian, F., et al. 2008, Astropart. Phys., 29, 55

Baltz, E. A., Briot, C., Salati, P., Taillet, R., \& Silk, J. 2000, Phys. Rev. D, 61, 023514

Bellazzini, M., Ferraro, F. R., \& Ibata, R. 2003, AJ, 125, 188

Belokurov, V., et al. 2006a, ApJ, 647, L111

Belokurov, V., et al. 2006b, ApJ, 642, L137

Belokurov, V., et al. 2007, ApJ, 654, 897

Binney, J., \& Tremaine, S. 2008, Galactic Dynamics (2nd ed.; Princeton, NJ: Princeton Univ. Press)

Bovill, M. S., \& Ricotti, M. 2008, arXiv:806.2340

Carraro, G., Zinn, R., \& Moni Bidin, C. 2007, A\&A, 466, 181

Casetti-Dinescu, D. I., Girard, T. M., Herrera, D., van Altena, W. F., López, C. E., \& Castillo, D. J. 2007, AJ, 134, 195

Chonis, T. S., \& Gaskell, C. M. 2008, AJ, 135, 264

Clem, J. L., Vanden Berg, D. A., \& Stetson, P. B. 2008, AJ, 135, 682

Driscoll, et al. (STACEE Collaboration) 2007, arXiv:0710.3545

Faber, S. M., et al. 2003, in Proc. SPIE 481 Instrument Design and Performance for Optical/Infrared Ground-based Telescopes ed. Iye \& Moorwood, (Bellingham, WA: SPIE), 1657

Fellhauer, M., et al. 2006, ApJ, 651, 167

Gilmore, G., Wilkinson, M. I., Wyse, R. F. G., Kleyna, J. T., Koch, A., Evans, N. W., \& Grebel, E. K. 2007, ApJ, 663, 948

Girardi, L., Grebel, E. K., Odenkirchen, M., \& Chiosi, C. 2004, A\&A, 422, 205

Harris, W. E. 1996, AJ, 112, 1487

Helmi, A., et al. 2006, ApJ, 651, L121

Illingworth, G. 1976, ApJ, 204, 73

Irwin, M., et al. 2007, ApJ, 656, L13

Johnston, K. V., Law, D. R., \& Majewski, S. R. 2005, ApJ, 619, 800

King, I. R. 1966, AJ, 71, 64

Kirby, E. N., Guhathakurta, P., \& Sneden, C. 2008a, ApJ, 682, 1217

Kirby, E. N., Simon, J. D., Geha, M., Guhathakurta, P., \& Frebel, A. 2008b, ApJ, 685,43

Kleyna, J. T., Wilkinson, M. I., Evans, N. W., \& Gilmore, G. 2005, ApJ, 630, L141

Klimentowski, J., Łokas, E. L., Kazantzidis, S., Prada, F., Mayer, L., \& Mamon, G. A. 2007, MNRAS, 378, 353

Koch, A., Grebel, E. K., Gilmore, G. F., Wyse, R. F. G., Kleyna, J. T., Harbeck, D. R., Wilkinson, M. I., \& Wyn Evans, N. 2008, AJ, 135, 1580

Koposov, S., et al. 2008, AJ, 686, 279

Law, D. R., Johnston, K. V., \& Majewski, S. R. 2005, ApJ, 619, 807

Madau, P., Diemand, J., \& Kuhlen, M. 2008, ApJ, 679, 1260

Majewski, S. R., Skrutskie, M. F., Weinberg, M. D., \& Ostheimer, J. C. 2003, ApJ, 599, 1082

Majewski, S. R., et al. 2004, AJ, 128, 245

Maraston, C. 2005, MNRAS, 362, 799

Martin, N. F., de Jong, J. T. A., \& Rix, H.-W. 2008, ApJ, 684, 1075

Martin, N. F., Ibata, R. A., Chapman, S. C., Irwin, M., \& Lewis, G. F. 2007, MNRAS, 380, 281

Mateo, M. L. 1998, ARA\&A, 36, 435

Mateo, M., Olszewski, E. W., Pryor, C., Welch, D. L., \& Fischer, P. 1993, AJ, 105,510

Mayer, L., Mastropietro, C., Wadsley, J., Stadel, J., \& Moore, B. 2006, MNRAS, 369, 1021

Muñoz, R. R., Carlin, J. L., Frinchaboy, P. M., Nidever, D. L., Majewski, S. R., \& Patterson, R. J. 2006, ApJ, 650, L51

Muñoz, R. R., Majewski, S. R., \& Johnston, K. V. 2008, ApJ, 679, 346

Olszewski, E. W., Pryor, C., \& Armandroff, T. E. 1996, AJ, 111, 750

Pritzl, B. J., Venn, K. A., \& Irwin, M. 2005, AJ, 130, 2140

Putman, M. E., Grcevich, J., \& Peek, J. E. G. 2008, arXiv:0803.306903

Ricotti, M., Gnedin, N. Y., \& Shull, J. M. 2008, arXiv:0802.2715

Rider, C. J., Tucker, D. L., Smith, J. A., Stoughton, C., Allam, S. S., \& Neilsen, Jr, E. H. 2004, AJ, 127, 2210

Ritz, S. M., Michelson, P. F., Meegan, C., Grindlay, J., \& GLAST Mission Team. 2007, in American Astronomical Society Meeting Abstracts, Vol. 211, 98.01 
Robin, A. C., Reylé, C., Derrière, S., \& Picaud, S. 2003, A\&A, 409, 523

Rutledge, G. A., Hesser, J. E., \& Stetson, P. B. 1997, PASP, 109, 907

Sakamoto, T., \& Hasegawa, T. 2006, ApJ, 653, L29

Schlegel, D. J., Finkbeiner, D. P., \& Davis, M. 1998, ApJ, 500, 525

Siegel, M. H., Shetrone, M. D., \& Irwin, M. 2008, AJ, 135, 2084

Simon, J. D., \& Geha, M. 2007, ApJ, 670, 313

Sohn, S. T., et al. 2007, ApJ, 663, 960

Strigari, L. E., Bullock, J. S., Kaplinghat, M., Simon, J. D., Geha, M., Willman, B., \& Walker, M. 2008a, Nature, in press, arXiv:0808.3772

Strigari, L. E., Koushiappas, S. M., Bullock, J. S., Kaplinghat, M., Simon, J. D., Geha, M., \& Willman, B. 2008b, ApJ, 678, 614
Tollerud, E. J., Bullock, J. S., Strigari, L. E., \& Willman, B. 2008, ApJ, 688, 277

Tyler, C. 2002, Phys. Rev., D, 66, 023509

Walker, M. G., Mateo, M., Olszewski, E. W., Bernstein, R., Wang, X., \& Woodroofe, M. 2006, AJ, 131, 2114

Walsh, S. M., Jerjen, H., \& Willman, B. 2007, ApJ, 662, L83

Walsh, S., Willman, B., \& Jerjen, H. 2009, AJ, 137, 450

Willman, B., et al. 2005a, AJ, 129, 2692

Willman, B., et al. 2005b, ApJ, 626, L85

Wood, M., et al. 2008, ApJ, 678, 594

Zucker, D. B., et al. 2006a, ApJ, 650, L41

Zucker, D. B., et al. 2006b, ApJ, 643, L103 